conocimiento de los plecópteros euholognatos (Plecoptera: Euholognatha) de la Sierra de Las Villas (Jaén, España). Boletín de la Sociedad Entomológica Aragonesa 41:453-454.

HERVIER, J. -1905-Bull. Acad. Int. Géogr. Bot. Le Mans 15: 1-32.

HERVIER, J. -1906-Bull. Acad. Int. Géogr. Bot. Le Mans 16: 201-232.

MARTÍNEZ ORTEGA, M.M., J.A SÁNCHEZ AGUDO y E. RICO (inédito) Veronica L. in S. Castroviejo et al., eds., Flora Iberica vol. XIII. Real Jardín Botánico, Madrid. [Borrador de la síntesis del género, disponible en www. rjb.csic.es]

MATEO SANZ, G. y J.M. MORENO VALDEOLIAS -2004- "Nuevos datos sobre la flora de la provincia de Cuenca, XX" Flora Montiberica 26: 3-6.

ROALES, J. -1997- Contribución al conocimiento de la flora de Sevilla I: novedades corológicas para la provincia. Lagascalia 20(1): 129-150.

TRIANO, E. -1998- Flora del Subbético cordobés. Catálogo, recursos y curiosidades. Ayto. de Rute, Diputación Prov. de Córdoba, Córdoba.

VALDÉS, B., S. TALAVERA y E. FERNÁNDEZGALIANO -1987- Flora Vascular de Andalucía Occidental. Ed. Ketres, Sevilla.

Dirección del autor. C/ Cristóbal Millán 22, C.P. 23300 Villacarrillo (Jaén, España).

ggonjur@mixmail.com

\title{
145. LITTORELLA UNIFLORA EN LAS LAGUNAS DE MOGUER (PARQUE NATURAL DE DOÑANA, SW PENINSULA IBÉRICA)
}

\section{Pablo GARCÍA MURILLO* y Manuela PALACIOS GONZÁLEZ}

Recibido el 27 de marzo de 2009, aceptado para su publicación el 29 de junio de 2009

Publicado "on line" en octubre de 2009

\section{Littorella uniflora in Moguer Pond Complex (Doñana Natural Park, SW Iberian Peninsula)}

Palabras clave. Littorella, plantas acuáticas, flora acuática, Andalucía, Doñana, Peninsula Ibérica.

Key words. Littorella, aquatic plants, aquatic flora, Donana, Andalusia, Iberian Peninsula.

Huelva. Moguer. Lagunas de Moguer. Pastizales higroturbosos en el borde de una laguna. 29SPB9914, 22/3/2009. P. García Murillo \& M. Palacios (SEV237734).

En el año 1993 aparecía una nota en la revista Lagascalia firmada por Hellmann \&
Hellmann donde se informaba sobre el hallazgo de Littorella uniflora (L.) Ascherson en una zona del manto eólico litoral onubense, en el SW de Andalucía. Dicho hallazgo resultaba de gran interés desde el punto de vista corológico, ya que ampliaba considerablemente el área 


$\begin{array}{ll}\text { Tamaño de las plantas } & 7-8 \mathrm{~cm} \\ \text { Longitud y anchura de hojas } & 40-92 \times 1.2-1.4 \mathrm{~mm} \\ \text { Pedicelos de flores maculinas } & 12.6-37.00 \mathrm{~mm} \\ \text { Sépalos flores masculinas } & 4.6-5 \times 1.6-1.8 \mathrm{~mm} \\ \text { Tubos de corolas de flores masculina } & 4.8-5.2 \mathrm{~mm} \\ \text { Lóbulos de corolas de flores masculinas } & 1.2-2 \mathrm{~mm} \\ \text { Anteras } & 28-30 \times 1.0-1.2 \mathrm{~mm} \\ \text { Filamentos } & 14.2-16 \mathrm{~mm} \\ \text { Sépalos flores femeninas } & 3.2-4.8 \times 0.5-0.8 \mathrm{~mm} \\ \text { Corola flores femeninas } & 3.8-4.3 \mathrm{~mm} \\ \text { (longitud total) } & 14-15 \mathrm{~mm} \\ \text { Estilos } & 2.0 \times 1.2 \mathrm{~mm} \\ \text { Aquenios } & \end{array}$

Tabla 1. Características morfológicas de las plantas recolectadas.

de distribución (hasta el sur de la Península Ibérica) de esta especie, propia del centro, norte y oeste de Europa, con localidades aisladas en el Mediterráneo y poco frecuente en la mayor parte de la Península.

En esos momentos la localidad más próxima publicada se localizaba en Campo de Calatrava (Ciudad Real; cf. Carrasco et al., 1986), y aunque posteriormente se han ido añadiendo citas que delimitan con más precisión los límites meridionales de esta especie en la Península Ibérica: Arroyo de la Luz, Malpartida, Navalmoral de la Mata, en Cáceres (Ladero et al., 1995 y Molina Abril, 1996); Cabezarados, Fernáncaballero y Piedrabuena, en Ciudad Real (Martín Blanco \& Carrasco, 1998 y Molina Abril, 1996); Finca de Alto Tienda y La Calzada de Oropesa, en Toledo (Molina Abril, 1996) y Costa Vicentina, en el Algarve portugés (Costa et al., 1998); todas ellas quedan a una distancia considerable de la localidad señalada en esta nota.

Sin embargo, y del mismo modo que había ocurrido con las plantas de la localidad levantina de Sinarcas (Fos et al., 2008), desde la publicación de la nota de Hellmann \& Hellmann (1993) no se habían vuelto a tener noticias de la presencia de $L$. uniflora en la zona; por esta razón en la revisión de Flora iberica (Pedrol, 2009) la provincia de Huelva aparece entre paréntesis. Así pues, el motivo de esta nota es indicar el redescubrimiento de L. uniflora en una localidad próxima a las indicadas por Hellmann \& Hellmann (1993) y confirmar la existencia de esta planta en territorio andaluz, tras 18 años de incertidumbre desde su recolección en 1991.

La población observada se localizó en el borde de una de las lagunas del complejo de las Lagunas de Moguer, en la zona oeste del Parque Natural de Doñana, y esta formada por plantas terrestres, la mayor parte de ellas en flor en el momento de la recolección. Las plantas crecían sobre un suelo arenonoso-higroturboso, muy húmedo y pudimos contar unas 45 rosetas. Las características morfológicas de las plantas observadas se indican en la tabla 1. Asimismo la comunidad en la que se encontró incluía Agrostis estolonifera L., Baldellia ranunculoides (L.) Parl., Illecebrum verticillatum L. y se ubicaba en 
la orilla de una laguna de aguas temporales muy dulces $(110 \mu \mathrm{S} / \mathrm{cm})$, en las que se desarrollaba abundantemente Miriophyllum alterniflorum DC., junto con Eleocharis multicaulis (Sm.) Desv. e Isoetes velatum A. Braun.

Teniendo en cuenta el tipo de hábitat donde observamos que crecían las plantas, la información aportada por Hellman \& Hellman (1993) y Fos et al. (2008), y la dificultad de localización de la planta, entendemos que su distribución en la zona del manto eólico litoral onubense debe estar bastante más extendida de lo que se pensó en un principio, ya que en las inmediaciones de la laguna donde se encontró $L$. uniflora, se sitúan varias decenas de lagunas con características similares. El problema reside en su localización. Resulta extremadamente difícil la identificación de estas plantas en estado vegetativo, cuando se encuentran formando parte de un pastizal cuyos elementos muestran una morfología semejante a Littorella, sólo en el momento de la floración (posiblemente fugaz) esta especie es reconocible.

L. uniflora, como ya se ha indicado, no es una planta frecuente en la Península Ibérica, donde la mayor parte de sus poblaciones se localizan de forma aislada en el cuadrante NW; por ello algunas comunidades autónomas: Castilla-La Mancha, Madrid, País Vasco o Valencia, la incluyen en sus catálogos de especies protegidas. Las localidades Onubenses indicadas por Hellman \& Hellman (1993) y en esta nota, son las más meridionales de Europa y las únicas conocidas de Andalucía. Asimismo en lugares donde era frecuente, como Holanda o Gran Bretaña [aunque según Preston \& Croft (1997) nunca fue abundante], debido a la eutrofización de las aguas y a la destrucción de los hábitat donde vive, se ha observado una importante disminución en sus poblaciones (Preston \& Croft, 1997; Roefols, 1983). En consecuencia, las comunidades de Littorella se encuentran incluidas en la Directiva 92/43/ CEE, de "Habitat", con la calificación de "Habitat de interés comunitario" [con código
NATURA 2000: 3110 Aguas oligotróficas con un contenido de minerales muy bajo de las llanuras arenosas (Littorelletalia uniflorae)], traspuesta al ordenamiento jurídico español mediante los Reales Decretos 1997/1995 y $1193 / 1998$. Por último, de acuerdo con lo expuesto anteriormente, y con la certeza de que esta especie se encuentra en territorio andaluz; proponemos que en un futuro Littorella uniflora (L.) Ascherson se incluya entre las especies consideradas en la Lista Roja de la Flora Vascular de Andalucía.

\section{BIBLIOGRAFÍA}

CARRASCO, M.A., VELAYOS, M. y S. CIRUJANO -1986-. Notas sobre higrófitos peninsulares: Plantas del Campo de Calatrava -Ciudad Real, España). Lazaroa 10: 261-264.

COSTA, J. C., LOUSÃ, M. \& ESPÍRITO-SANTO, M. D. -1998-. Littorella uniflora (L.) Ascherson no Costeiro Vicentino, nova área de distribuição em Portugal continental. In Notas do Herbário da Estação Florestal Nacional (LISFA): Fasc. VIII. Silva Lusitana 6 (2): 259.

FOS MARTÍN, S., C. PEÑA BRETÓN, A. SEBASTIÁN DE LA CRUZ y V.I. DEL TORO TORRÓ -2008-. Redescubrimiento de Littorella uniflora en los lavajos de Sinarcas (Valencia). Flora Montiberica 39: 46-49.

HELLMANN, R. \& V. HELLMANN -1993-. Pilularia minuta Durieu (Marsileaceae) and Littorella uniflora (L.) Ascherson (Plantaginaceae) in South West Andalusia. Lagascalia 17: 189-190.

LADERO, M., AMOR, A., PÉREZ CHISCANO,J. L. \& M.T. SANTOS -1995-. Algunas plantas interesantes de la flora extremeña. Stud. Bot. Univ. Salamanca 14: 203-206.

MARTÍN BLANCO, C. J. y M. A. CARRASCO -1998-. Flora vascular del sector meridional de Montes Norte (Ciudad Real). Instituto de Estudios Manchegos. C.S.I.C.

MOLINA ABRIL, J.A. -1996-. De Hydrophytis Hispaniae Centralis Notulae Praecipue Chorologicae, II. Stud. Bot. Univ. Salamanca 15: 5-24.

PEDROL, J. -2008-. Littorella P. J. Bergius, In 
C. Benedi, E. Rico, J. Guemes \& A. Herrero (Eds.) Flora Ibérica. Vol. XIII. CSIC. Madrid. pp. 38-40.

PRESTON, C.D. \& J.M. CROFT -1997-. Aquatic plants in Britain and Ireland. Hartley Books. Colchester.

ROEFOLS, J.G.M. -1983-. Impact of acidification and eutrophication on macrophyte communities in soft waters in the Netherlands. I. Field observations. Aquatic Botany 17: 139-155.

Dirección de los autores. Departamento de Biología Vegetal y Ecología. Facultad de Farmacia. Universidad de Sevilla. C/. Profesor García González s/n. 41012 Sevilla. *Autor para correspondencia: pgarcia@us.es

\title{
146. NARCISSUS CAVANILLESII EN LA CUENCA DEL GUADIANA: HACIA UNA CONSERVACIÓN TRANSFRONTERIZA
}

\author{
Isabel MARQUES ${ }^{1 *}$, Antonia ROSSELLÓ-GRAELL ${ }^{1} \&$ David DRAPER $^{2}$
}

Recibido el 22 de Octubre de 2008, aceptado para su publicación el 2 de enero 2009 Publicado "on line" en marzo de 2009

\section{Trans-boundary conservation of Narcissus cavanillesii in the Guadiana valley}

Palabras clave. Fragmentación de poblaciones, comportamiento metapoblacional, conservación transfronteriza, Portugal, España.

Key words. Fragmented populations, metapopulational behavior, trans-boundary conservation, Portugal, Spain.

Narcissus cavanillesii A. Barra \& G. López (Amaryllidaceae) es una especie endémica del sur de la Península Ibérica y norte de África. Se trata de un pequeño geófito de floración otoñal contemplado en los Anexos II y IV de la Directiva Hábitat 92/43/CEE y en el Anexo 2 de la Ley 42/2007, de 13 de diciembre, del Patrimonio Natural y de la Biodiversidad (bajo el nombre de $N$. humilis).

En la Península Ibérica, el área de distribución de $N$. cavanillesii se restringe al cuadrante sudoeste, estando referenciada para la provincia portuguesa de Alto Alentejo (Malato-Beliz, 1977; Rosselló-Graell et al., 2003a) e para las provincias españolas de Cádiz, Córdoba, Huelva, Sevilla (Valdés et al., 1987), Málaga (MA 22495) y Badajoz (Devesa, 1995; Rivas Goday \& Ladero Álvarez, 1973). La cita para la provincia de Cáceres no debe de ser considerada pues se trata de error de identificación, ya confirmado por Barra (2002). Aunque hasta el momento no se conoce la 